\title{
Implementation and Test Results of a Chip for The Separation of Mixed Signals *
}

\author{
Ammar B. A. Gharbi and Fathi M. A. Salam \\ Circuits, Systems and Artificial Neural Networks Laboratory \\ Department of Electrical Engineering \\ Michigan State University, East Lansing, MI 48824 \\ email: gharbi@ee.msu.edu, salam@ee.msu.edu
}

\begin{abstract}
We describe an algorithm and chip implementation for separating a mixture of unknown, but independent, temporal signals in static and dynamic environments. The proposed algorithm, which is a simple modification of the Herault and Jutten (HJ) algorithm, proved to be robust to parameter variations. Moreover, we present some chip results to quantify the performance of the modified algorithm in static and dynamic (mixing and filtering) environments.
\end{abstract}

\section{INTRODUCTION}

The Separation of Independent Sources (SIS) assumes that some unknown but independent temporal signals propagate through a mixing and/or filtering natural or synthetic medium. By sensing outputs of this medium, a neural network is tailored to adaptively recover the original independent signals. Using only the property of independence, the neural network would work to counteract the effect of the mixing medium, see [1], [2], [3] and [4]. (More motivation of the potential uses of this approach are relegated to the cited references.) This work introduces a modification of the Herault-Jutten (HJ) algorithm which aspires to address the effect of dynamic media in filtering and delaying signals. It describes the implementation and application performance of a prototype neuro-chip in static and $d y$ namic environments, and experimentally quantifies the chip's capability and limitation. The paper is organized as follows. Section 2 defines the problem of separation of independent sources and summarizes the HJ algorithm. Section 3 describes the modification of the algorithm and its motivation. Section 4 presents the basic building blocks used to implement the proposed algorithm. Section 5 summarizes

* Acknowledgment: This work is supported in part by the Michigan Research Excellence Fund (REF). the experimental results of the chip testing. Some concluding remarks are given in section 6 .

\section{PROBLEM DEFINITION}

The general block diagram for SIS is shown in Figure 1 . The vectors $s(t), e(t)$, and $y(t)$ are, respectively, the unknown source vector, the measured signal vector, and the output signal vector. The network to be designed receives the signal $e(t)$ and adaptively modifies $y(t)$ to reproduce the original signal $s(t)$.

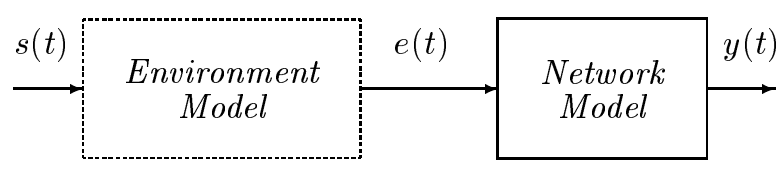

Figure 1. Block diagram

In [1] and [2], Herault and Jutten had proposed an algorithm that assumes a linear medium with no delays. The input to the network model is a measured signal vector, $\mathrm{e}(\mathrm{t})$ :

$$
e(t)=A s(t)
$$

where $A$ is a matrix whose components are all positive and which models the environment statically. Furthermore, $A$ is assumed to be nonsingular. Its diagonal entries are all ones and each off diagonal element is less than one. Herault and Jutten [1,2] used a recursive architecture made up of fully interconnected outputs. Each output, $y_{i}(t)$, receives the mixed signal, $e_{i}(t)$, and a weighted sum of all other outputs, $-\sum_{j \neq i} d_{i j} y_{j}(t)$. Thus,

$$
y_{i}(t)=e_{i}(t)-\sum_{j \neq i} d_{i j} y_{j}(t) \quad 1 \leq i \leq n
$$

which, in matrix form, becomes the (static) network:

$$
y(t)=e(t)-D y(t)
$$

where $\mathrm{D}$ is an $n \times n$ matrix whose main diagonal is zero. Now, the problem of separation of signals 
translates to retrieving the original signals. In the limit, it is thus desired to have:

$$
y(t)=P s(t)
$$

where $\mathrm{P}$ is a permutation matrix ( i.e. a matrix obtained from the identity by row and/or column permutation). From biological and intuitive inspirations, Herault and Jutten [1, 2] proposed the following update law:

$$
\dot{d}_{i j}=\eta_{i j} f\left(y_{i}\right) g\left(y_{j}\right)
$$

where $f($.$) and g($.$) are two nonlinear odd functions$ and $\eta_{i j}$ is the learning rate. This algorithm has been implemented in CMOS. Successful testing of several implementations, using static models of the environment and the network, described by (1) and (2), has been reported in [5] and [6]. However, the algorithm, and thus its implementations, lacks robustness to changes in the environment model.

\section{A MODIFIED HJ ALGORITHM}

The network of (2) can be rewritten as

$$
0=-y_{i}+e_{i}-\sum_{j \neq i} d_{i j} y_{j}
$$

To include the effect of the transient dynamics, we consider the dynamic network

$$
\tau_{i} \dot{y}_{i}(t)=-y_{i}(t)+e_{i}(t)-\sum_{j \neq i} d_{i j} y_{j}(t)
$$

where $\tau_{i}$, for all $\mathrm{i}$, is selected to provide time-scale separation between (5) and (3). By considering the dynamic network (5) in conjunction with the update law (3), only the stable and robust solutions of (5) become feasible solutions. This robustness property is important in lieu of the fact that physical circuit realization is necessarily prone to function approximation and inaccuracies. Moreover, this modified algorithm performs smoothing and enables an integrated circuit implementation to dominate the everpresent parasitic capacitance of its transistors. In [4], simulation results had shown that the system described by (1), (3) and (5) successfully separates mixed signals more robustly. Our chip implementation of system (3) and (5), discussed below, will be tested in both the static environment case as well as the more realistic dynamic environment case.

\section{VLSI IMPLEMENTATION}

The modified HJ algorithm is implemented in CMOS using $2.0 \mu \mathrm{m}$ technology on a Tiny Chip $(2.22 \times$ $2.25 \mathrm{~mm}^{2}$.) Similar building blocks to that of the Vittoz-Arreguit implementation are used. The basic components of the circuit are a transconductance amplifier [7] and a sine hyperbolic circuit [5]. In Figure 2, the circuit diagrams of the basic components and their corresponding measured characteristics are shown. These basic components produce a relationship between the input voltage $\mathrm{V}$ and the output current, which is expressed in units of $\frac{k T}{q}$ (see [7]):

$$
\begin{aligned}
I_{\text {out }}^{\text {tanh }} & =I_{b} \tanh \frac{\kappa}{2} V \\
I_{\text {out }}^{\text {sinh }} & =2 w I_{0} e^{\kappa\left(V_{b}+\delta V\right)} \sinh \kappa V
\end{aligned}
$$

It is important to observe that some of the parameters that the above currents depend on are: the biasing voltage $V_{b}$; the ratio of width of the biasing transistor to the width of the arm transistors $w$; and the offset voltage $\delta V$. Equations (3) and (5) are implemented in CMOS by the circuit diagrams shown in Figure 4:

$$
\begin{array}{r}
\dot{V}_{i j}=\frac{2 w I_{0} e^{\kappa\left(V_{b}+\delta V\right)}}{C_{i j}} \tanh \frac{\kappa}{2} V \sinh \kappa V \\
\frac{\bar{C}_{i}}{\beta\left(V_{R}-V_{T}\right)} \dot{y}_{i}=e_{i}-y_{i}+\sum_{j \neq i} \frac{V_{i j}-V_{T}}{V_{R}-V_{T}} y_{i}
\end{array}
$$

The table below shows the parameters in equations (3) and (5), their corresponding CMOS expressions as well as their nominal values in the course of testing.

\begin{tabular}{||l|c|c||}
\hline & CMOS expression & Nominal value \\
\hline$\tau_{i}$ & $\frac{C_{i}}{\beta\left(V_{R}-V_{T}\right)}$ & $1.2 \times 10^{-8} s$ \\
$\eta_{i j}$ & $\frac{2 w I_{0} e^{\kappa\left(V_{b}+\delta V\right)}}{C_{i j}}$ & $8.0 \times 10^{4} s^{-1}$ \\
\hline
\end{tabular}

Using these basic building blocks, the circuit implementation of our algorithm, governed by (3) and (5), is directly realized. Observe that the ratio between $\tau_{i}$ and the inverse of $\eta_{i j}$ is about 4-folds, leading to a fast and slow time-scales of the dynamics of the network and the weight update.

\section{TEST RESULTS}

The resulting chip is tested to quantify its performance for static and dynamic media.

\subsection{The Static Medium Case}

It is assumed here that the mixed signals are linear combinations of the unknown sources as described in (1). The chip testing has been carried out using a two-neuron network where it is desired to separate two independent signals.

\subsubsection{The Experimental Setup}

The combined circuits shown in Figure 5, with $C_{F i j}=0$, will produce a linear combination of the original sources. Summing all the currents at node 1 in Figure 5(b) gives

$$
e_{i}=\sum_{j=1}^{n} \frac{R_{0}}{R_{i j}} s_{j}=s_{i}+\sum_{j \neq i} \frac{R_{0}}{R_{i j}} s_{j}
$$

since $R_{i i}=R_{0}=10 k \Omega$. Thus, the coefficient of the matrix $\mathrm{A}$ in (1), $a_{i j}=\frac{R_{0}}{R_{i j}}$, can be varied by using 
an external variable potentiometer for $R_{i j}$. Such variations can be used to study the robustness of the network realized on the Tiny Chip.

\subsubsection{Experimental Results}

Experiments were conducted to investigate the performance of the chip for the separation of the signals in three scenarios: (i) separation of two segments of music signals (Japanese and English) from two different sources, (ii) separation of two speech segments of two different English speakers, and (iii) separation of white noise and an English speech segment. Successful separation was obtained in the first two cases for mixing levels less than $80 \%$. For the third case, perfect separation is attained only for mixing levels less than 30\%. See Figure 3. Above these levels, the outputs of the network are not separated but rather contain a mixture of the original signals.

For the three mentioned scenarios, the level of mixing has been varied in order to study the robustness of the network to variations. For this reason, the parameters $a_{i j}$ are initially fixed to some values. Once the network converged (and separation of signals is established), the parameters $a_{i j}$ are then varied in order to observe the quality of performance. Experiments for different starting values of the mixing matrix $A$ were performed. The percentage of the robustness to each parameter is then recorded. Based on the experiments, the network is robust to parameter variations of about $15 \%$ (on average).

\subsubsection{Limitations}

As the level of mixing is varied, it was discovered that the network fails to separate the signals when the mixing reaches some level. Based on experiments, the coefficients of the mixing matrix $\mathrm{A}$ should range between 0.0 and 0.8 for the first two scenarios and 0.0 and 0.3 for the third scenario.

\subsection{The Dynamic Modeling Case}

It is now assumed that the input to the network is a superposition and a delayed (filtered) version of the unknown sources. This is a more realistic, realworld, mixing scenario.

\subsubsection{Experimental Setup}

The circuit diagrams shown in Figure 5 (a) is used to obtain a filtered version of the original signals:

$$
e_{i}=\sum_{j=1}^{n} F_{i j}\left(s_{j}\right)
$$

$F_{i j}($.$) is a low pass filter with gain g_{i j}$ and cutoff frequency $\omega_{i j}$.

$$
\begin{aligned}
& F_{i j}\left(V_{i n}\right)=\frac{g_{i j}}{\frac{s}{\omega_{c i j}}+1} V_{i n} \\
& g_{i j}=\frac{R_{0}}{R_{i j}} \quad \omega_{c i j}=\frac{1}{R_{0} C_{F i j}}
\end{aligned}
$$

\subsubsection{Experimental Results}

The goal now is to study whether the modified algorithm can still update the parameters where the parameters $d_{i j}$ will adaptively counter the effect of $g_{i j}$ and $\omega_{c i j}$, and thus recover the original signals. The three previous scenarios are repeated in studying this problem. Exhaustive experimentation and testing led to the conclusion that separation of the signals occurs in the first two scenarios when $g_{i j}<0.4$ and also in the third scenario when $g_{i j}<0.15$. See Figure 6 for example results.

\subsubsection{Limitations}

It is noted that the interval range over which the separation occured had shrunk considerably in comparison to the linear static medium case. Nonetheless, the chip was able to achieve signal separation in this dynamic medium as well.

\section{CONCLUSION}

We have tested chip implementation of the modified HJ algorithm and explored its validity range. The modification produces robust performance to parameter variations and to dynamic media effects. Limits were also experimentally quantified. The fact that realistic sensors have their own inherent dynamics underlines the need for such consideration. We showed that the modified algorithm has a potential in solving the problem of SIS in more realistic realworld environments. Despite success in our reported cases, however, there are still challenging problems ahead for full practical use.

\section{REFERENCES}

[1] J. Herault and C. Jutten. Blind separation of sources, part 1: An adaptive algorithm based on neuromimetic architecture. Signal Processing, 24:1-10, July 1991.

[2] J. Herault and C. Jutten. Blind separation of sources, part 2: Problem statement. Signal Processing, 24:11-20, July 1991.

[3] J. C. Platt and F. Faggin. Networks for the separation sources that are superimposed and delayed. Advances in Neural Information Processing systems, 1:730-737, 1992.

[4] F. M. A. Salam. An adaptive network for blind separation of independent signals. International Symposium on Circuits and Systems, 1:431-434, May 1993.

[5] E. Vittoz and X. Arreguit. Cmos integration of herault-jutten cells for separation of sources. Proceedings Workshop on Analog VLSI and Neural Systems, May 1989.

[6] M. H. Cohen and G. Andreou. Current-mode subthreshold mos implementation of heraultjutten autoadaptive network. IEEE Journal of Solid-State Circuits, 27(5):714-727, May 1992.

[7] C. Mead. Analog VLSI and Neural Systems. Prentice Hall, New York, 1989. 


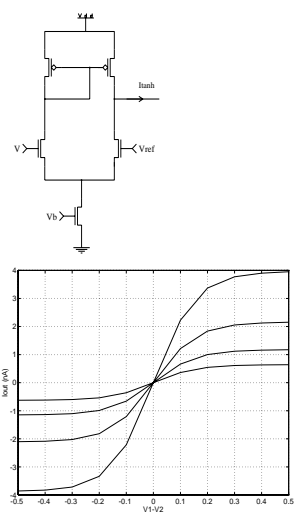

Hyperbolic tangent
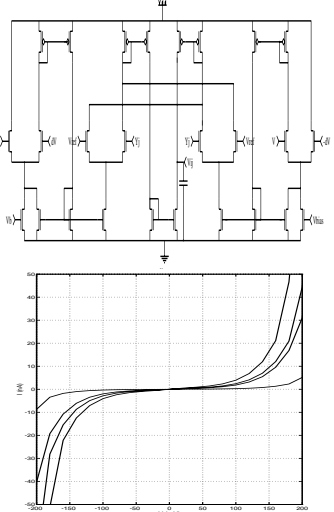

Hyperbolic sine

Figure 2. Basic Analog Components
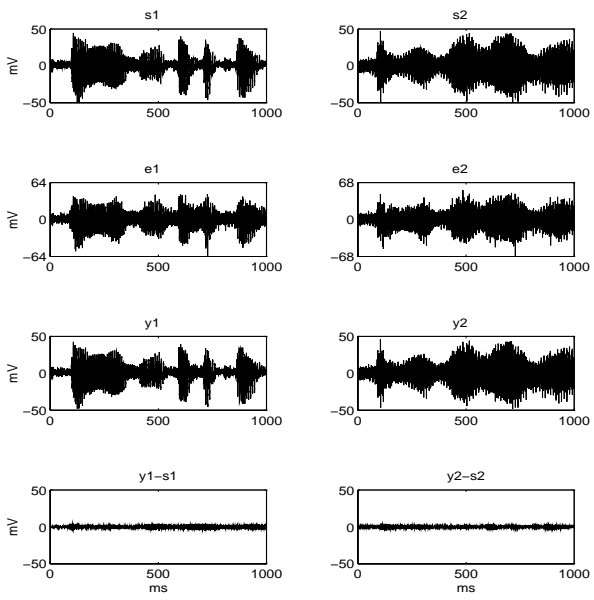

(a) Japanese and English segments
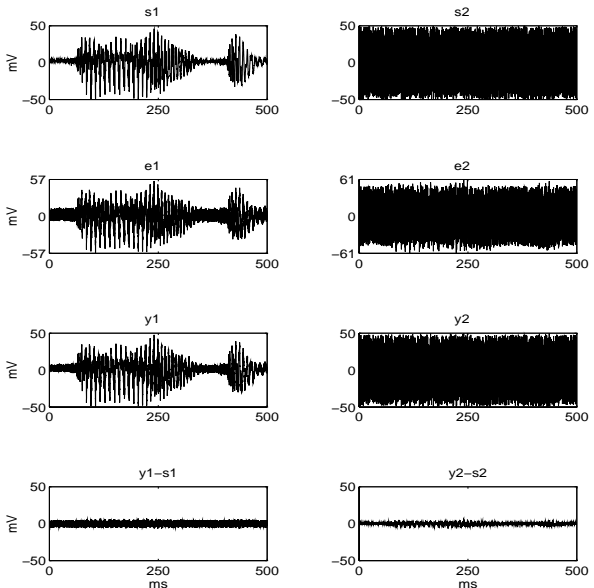

(b) White signal and English segment

Figure 3. Static Mixing

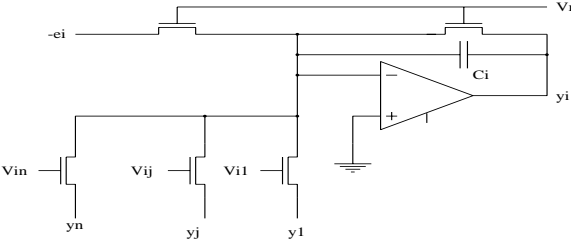

Figure 4. Summing Unit

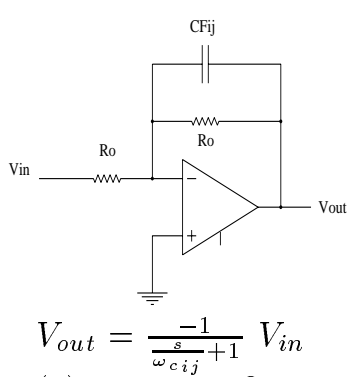

(a) Low pass filter

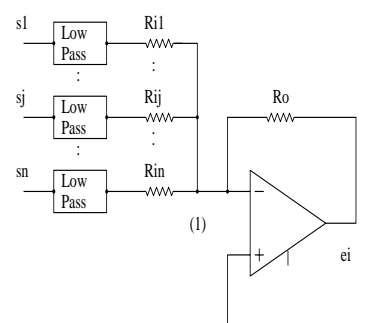

$e_{i}=\sum_{j=i}^{n} \overline{\overline{\bar{F}_{i j}}}\left(s_{j}\right)$

(b) Mixing/filtering circuit
Figure 5. Mixing Circuit
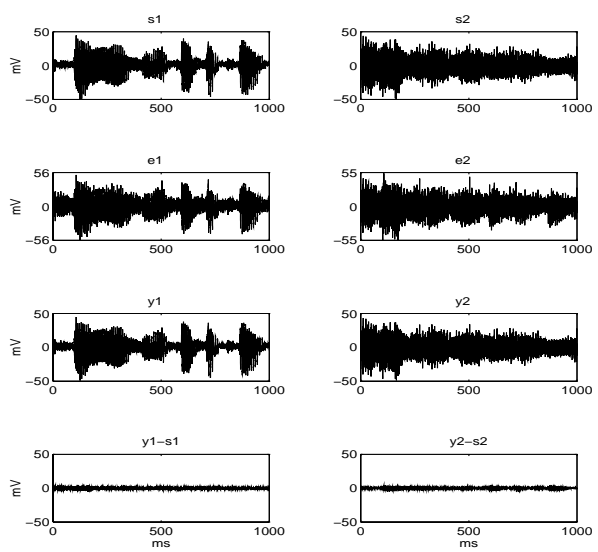

(a) Japanese and English segments
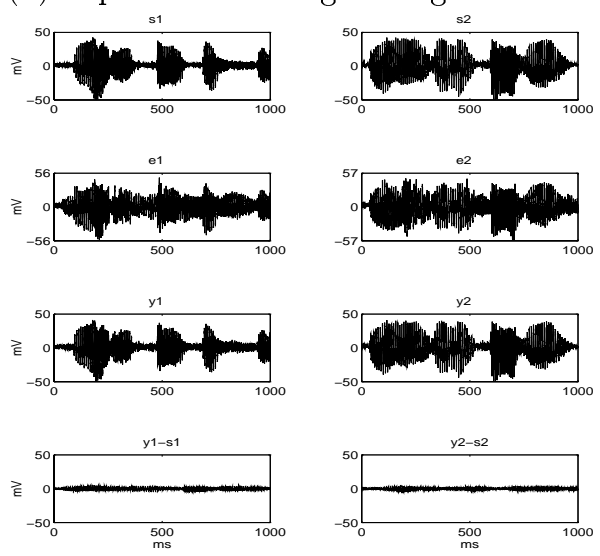

(b) Both English segments

Figure 6. Dynamic Mixing 